\title{
Creating a FACETS digital toolkit to promote quality of life of people with multiple sclerosis through participatory design
}

\author{
Beth Fairbanks, Huseyin Dogan, Nan Jiang, Keith Pretty \\ Faculty of Science and Technology \\ Bournemouth University \\ \{i7670465, hdogan, njiang, kpretty\}@bournemouth.ac.uk
}

\author{
Andy Pulman, Peter Thomas, Sarah Thomas \\ Faculty of Health and Social Sciences \\ Bournemouth University \\ \{apulman, pthomas, saraht\}@bournemouth.ac.uk
}

\begin{abstract}
In this paper, we report on the first stages of creating a stand-alone digital toolkit focusing on the homework elements of FACETS (Fatigue: Applying Cognitive behavioural and Energy effectiveness Techniques to lifeStyle). FACETS is an evidence-based face-to-face fatigue management group programme for people with multiple sclerosis. This paper details the participatory design process from requirements elicitation to initial prototyping and how offline activities linked to each session have been mapped in the digitised solution (mobile app).
\end{abstract}

FACETS, Multiple Sclerosis, Fatigue, Cognitive Behavioural Model (CBM), Digital Health, Participatory Design.

\section{INTRODUCTION}

Multiple Sclerosis (MS) is a neurological condition affecting the central nervous system. Over 2.5 million people have MS worldwide (Kanavos et al. 2016). Fatigue is one of the most common and debilitating symptoms of MS. It is the main reason why people with MS (pwMS) stop working early and its invisible nature can make it difficult to understand and recognise.

\section{FACETS}

FACETS is a group-based fatigue management programme for pwMS developed by a team at the Bournemouth University Clinical Research Unit (BUCRU) along with collaborators from Poole Hospital. The programme format incorporates short presentations, group activities and discussions, and homework tasks designed to provide attendees with skills to help them to make changes to their lifestyle and in attitudes to managing their fatigue. The programme blends principles of energy effectiveness with Cognitive Behavioural (CB) approaches (Thomas S., et al. 2010) and is offered in six weekly sessions with refreshment breaks, facilitated in groups of $6-12$ by two healthcare professionals (HCPs).

FACETS has been evaluated in an MS Society funded trial. Participants were randomised into two groups; one of which attended the FACETS programme in addition to usual care, and one of which continued with their routine care. The results showed that, relative to the control group, the FACETS group demonstrated improvements in fatigue severity and self-efficacy at a four-month follow-up. A year on from the beginning of the trial, improvements were still sustained and additional improvements in quality of life were emerging (Thomas PW., et al. 2010; Thomas S., et al. 2013; Thomas PW., et al. 2014).

\subsection{Aim and Structure}

The aim of FACETS is to support pwMS to understand more about MS fatigue and to normalise their fatigue experiences, learn helpful ways of thinking about fatigue and use available energy more effectively (Thomas S., et al. 2010).

The structure of each FACETS session allows for personal reflections, discussion with trusted others in similar positions and the opportunity to think about and make behavioural and attitudinal changes which will potentially have an impact beyond the duration of the programme. A paced delivery over six weeks allows for adequate breaks between sessions, an opportunity to reflect on topics covered, and to undertake the set homework activities. An overview of the sessions is provided in Table 1. 
Table 1: Overview of FACETS

\begin{tabular}{|l|l|}
\hline Session & \multicolumn{1}{c|}{ Title } \\
\hline 1 & What is MS-related fatigue? \\
\hline 2 & Opening an 'energy account' \\
\hline 3 & $\begin{array}{l}\text { Budgeting energy and 'smartening up' } \\
\text { goals }\end{array}$ \\
\hline 4 & $\begin{array}{l}\text { The stress response; the cognitive } \\
\text { behavioural model }\end{array}$ \\
\hline 5 & $\begin{array}{l}\text { Putting unhelpful thoughts on trial } \\
\text { Recapping and taking the programme } \\
\text { forward }\end{array}$ \\
\hline 6 & \multicolumn{1}{|c|}{} \\
\hline
\end{tabular}

\subsection{Key Aspects of FACETS: Communication and Group Dynamic}

The group dynamic aspect of FACETS is considered fundamental to the success of the programme and has been described by healthcare professionals (HCPs) and pwMS as being the most important element (Pulman A., et al. 2018). The group provides an environment for peer learning and sharing where empathy, mutual support, and sometimes plain speaking can be of huge benefit to attendees.

\subsection{Key Aspects of FACETS: Cognitive Behavioural Model}

The FACETS programme is based upon a conceptual framework that integrates cognitive behavioural, social cognitive and energy effectiveness theories and principles. FACETS aims to support people with MS to self-manage their fatigue by providing them with tools and strategies that they can apply to their daily lives (Thomas S., et al. 2010). In the programme there is a gradual transition from a practical to a more psychological orientation. However, cognitive behavioural (CB) elements are introduced early, enabling group members to explore reciprocal links between the CB components (behaviours, emotions, thoughts and physical aspects), before the Cognitive Behavioural Model (CBM) is formally introduced in Session 4.

\subsection{Homework}

Homework tasks are an integral part of the FACETS programme. Each week attendees are asked to complete a short task designed to help them put into practice some of the new skills and strategies they have learned. Group members are strongly encouraged to complete the homework tasks. A summary of homework for each session is provided in Table 2.
Table 2: Overview of FACETS homework

\begin{tabular}{|l|l|}
\hline Session & \multicolumn{1}{c|}{ Homework } \\
\hline 1 & Activity/fatigue diary; Energy measure \\
\hline 2 & Rest/activity/sleep planner \\
\hline 3 & Goal-setting exercise \\
\hline 4 & Fatigue thought diary \\
\hline 5 & Thought challenge sheet \\
\hline 6 & 'Keeping on Track' planner \\
\hline
\end{tabular}

\subsection{Pathway to Digital Toolkit}

The MS Society commissioned our team to undertake a consultation to gather the views of pwMS and HCPs regarding an online model of service delivery and how FACETS could be best adapted into a digitised format (known as 'CFACETS') (Pulman A., et al. 2018). Based on data collected and a roundtable discussion, a minimal viable product of cFACETS was deemed the best starting point - enabling a solution that could then be added to over time. A key recommendation from the consultation was that a separate project should look into creating a free stand-alone digital toolkit focusing on the homework elements of FACETS (see Table 2).

\section{Requirements elicitation}

Data from the cFACETS consultation (Pulman A., et al. 2018) informed the initial set of draft requirements which were then categorised using the MoSCoW method (Clegg and Barker 1994) for prioritisation. The following were the 'must-have' requirements for the toolkit:

- Allow the user to complete the homework tasks and set goals.

- Allow personalisation/flexibility to the user's needs.

- Save progress to the cloud.

- Give the user an option to print blank or completed homework worksheets.

- Provide user prompts and hints to complete tasks (e.g. example answers and tutorials for further explanation).

- Incorporate intuitive user interfaces for easy navigation.

- Encrypt all entered data.

- Give the user the option of storing data on the cloud or local storage.

- Save progress every two seconds and allow the user to save and exit to come back later.

- Timestamp all diary entries.

- Notify the user when a new update is available, as they open the toolkit. 
- Track overall energy levels and daytime napping duration.

- Track fatigue.

- Work across multiple devices.

- Allow the user to view and edit previous entries.

\section{DESIGN ITERATIONS}

\subsection{Participatory design (PD) participant profiles}

Participants comprised core members of the project team and potential end users. One potential user involved with the project was a British male, aged 59, diagnosed with Secondary Progressive MS in 2008. The other was also a British male, aged 53, diagnosed with Secondary Progressive MS in 2016. Table 3 gives an overview of design team members.

Table 3: Overview of Participants

\begin{tabular}{|l|l|l|l|}
\hline Member & Gender & \multicolumn{1}{|c|}{$\begin{array}{c}\text { Expertise/Key } \\
\text { Characters }\end{array}$} & \multicolumn{1}{|c|}{$\begin{array}{c}\text { User } \\
\text { Group }\end{array}$} \\
\hline$\# 1$ & Female & $\begin{array}{l}\text { Healthcare; } \\
\text { Psychology; } \\
\text { Behaviour change }\end{array}$ & $\begin{array}{l}\text { Project } \\
\text { team }\end{array}$ \\
\hline$\# 2$ & Male & Digital & $\begin{array}{l}\text { Project } \\
\text { team }\end{array}$ \\
\hline$\# 3$ & Male & Digital & $\begin{array}{l}\text { Project } \\
\text { team }\end{array}$ \\
\hline$\# 4$ & Female & $\begin{array}{l}\text { Healthcare; } \\
\text { qualitative }\end{array}$ & $\begin{array}{l}\text { Project } \\
\text { team } \\
\text { Post Doc }\end{array}$ \\
\hline$\# 5$ & Male & Digital; Healthcare & $\begin{array}{l}\text { Project } \\
\text { team } \\
\text { Post Doc }\end{array}$ \\
\hline$\# 6$ & Female & Carer & End user \\
\hline$\# 7$ & Male & pwMS & End user \\
\hline$\# 8$ & Male & pwMS; Digital & End user \\
\hline$\# 9$ & Male & $\begin{array}{l}\text { Clinical statistics; } \\
\text { Carer }\end{array}$ & $\begin{array}{l}\text { Project } \\
\text { Team }\end{array}$ \\
\hline
\end{tabular}

\subsection{Iterations}

One workshop and three interviews were conducted to gather initial requirements about client expectations regarding digitisation of the toolkit. Three bi-weekly meetings were then held with the project team to discuss and decide on progress, undertake a card sorting task and requirements prioritisation. At each meeting, revised versions of screen designs were presented for comment and discussion from both a technical and health perspective, bearing in mind the particular needs of the users and the structure and methodology underpinning the original programme.

\subsection{Mapping of the homework elements}

All pages have access to the sidebar menu, progress bar and accessibility functionality e.g. text-to-speech and speech-to-text. Table 4 presents mappings between the homework elements and the digital features and functionalities of the digitised element.

Table 4: Homework Element Features

\begin{tabular}{|l|l|}
\hline Homework Element & Features/ Functionalities \\
\hline Activity/fatigue diary & $\begin{array}{l}\text { Optional hourly reminders to } \\
\text { complete this; symptom } \\
\text { logging; fatigue diary uses the } \\
\text { activities entered in activity } \\
\text { diary for ratings. }\end{array}$ \\
\hline $\begin{array}{l}\text { Rest/activity/sleep } \\
\text { planner }\end{array}$ & $\begin{array}{l}\text { Automated/ manual sleep } \\
\text { tracking; set number of rests } \\
\text { for the next day; view sleep } \\
\text { graph if use a phone/watch } \\
\text { sleep sensor. }\end{array}$ \\
\hline Goal-setting exercise & $\begin{array}{l}\text { Select two lifestyle changes to } \\
\text { make; fit them into your } \\
\text { routine; create SMART goals. } \\
\text { Indicate goal achievement } \\
\text { when these have been } \\
\text { completed. }\end{array}$ \\
\hline Fatigue thought diary & $\begin{array}{l}\text { Write down unhelpful fatigue- } \\
\text { related thoughts along with } \\
\text { trigger situation and } \\
\text { accompanying emotions. }\end{array}$ \\
\hline $\begin{array}{l}\text { Rating unhelpful fatigue- } \\
\text { related thoughts and } \\
\text { associated emotions and } \\
\text { coming up with alternative } \\
\text { thoughts; re-rating the original } \\
\text { unhelpful thoughts and } \\
\text { emotions. }\end{array}$ \\
\hline $\begin{array}{l}\text { Selecting priority areas for } \\
\text { managing fatigue over the next } \\
\text { 3 months; making plans to } \\
\text { work towards these by setting } \\
\text { goals and creating coping } \\
\text { plans. }\end{array}$ \\
\hline
\end{tabular}

\subsection{Refined designs}

The user can sign in with their account details; create a new account; sign in with Facebook or via a cloud platform. Figure 1 shows the dashboard and 'adding a homework element' screens (e.g. activity, rest time, goal, mood and future plans). The user is given an option to view completed activities, print, view/edit goals, change settings etc. 


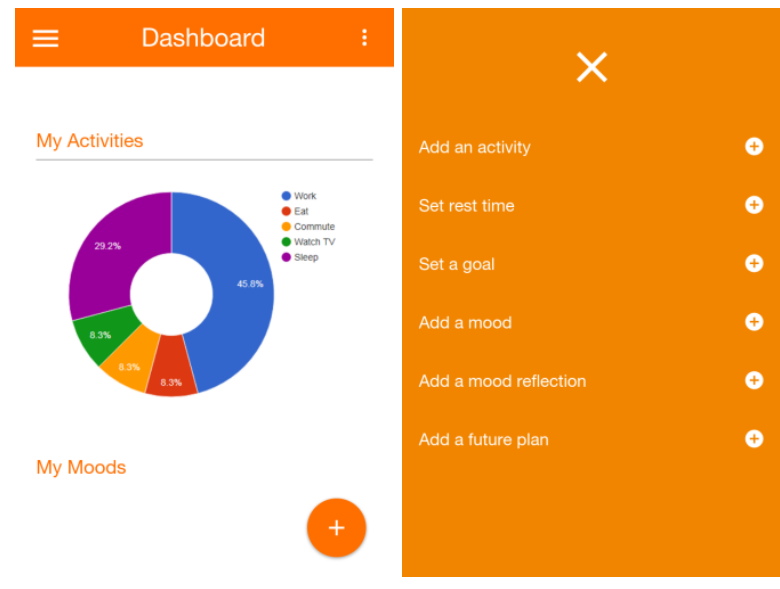

Figure 1: Dashboard and homework elements

\section{LESSONS LEARNT}

A prototype of the digital fatigue management toolkit based on FACETS was successfully produced by taking the PD approach. Through practising $\mathrm{PD}$, the following lessons were learnt:

- The recruitment of appropriate participants with a good and balanced representation of health, digital, service user and carers was essential to the successful practice of PD. In particular, the involvement of end users with MS (including one person with a digital background) has helped to contribute to architectural design and improvements from a user's perspective. The comments made by participating carers also provided helpful insights into how the digital toolkit could be used.

- Having face-to-face meetings proved very beneficial for determining the information architecture of the digital toolkit and providing constructive feedback on the design iterations. However, organising such meetings was a challenging task due to factors such as team members' availability as well as accessibility issues. This suggests that face-to-face meetings with remote support or remote meetings could be considered in the future.

- We used an online based interactive prototyping tool for the design iterations. We found that the interactive prototype helped us envision our ideas more effectively than with paper prototyping. It also helped address some of the challenges of working across digital and health fields. This suggests that, if possible, an interactive prototyping tool such as InVision or Proto.io should be considered to maximise the advantages of PD.
- The digital toolkit was developed based on the homework elements of FACETS but it did not provide an exact one-to-one mapping to the elements mainly due to the purpose of the toolkit. Moreover, after analysing the paper handouts and discussions with the FACETS team, it was found that some homework elements could be consolidated into a single entity in the digital version. This suggests that when considering the digitisation of a paperbased solution, we need to consider the availability of the technologies as well as the shift from paper medium to digital medium.

\section{CONCLUSIONS}

A digital toolkit will add value to cFACETS and will bridge a gap where no such clinically proven solutions currently exist for pwMS. These might encompass a suite of mobile applications including a number of homework elements from the existing programme in addition to real-time symptom loggers. Further consideration should be given to the unique requirements of the end user and the importance of their meaningful involvement in the development, prototyping and piloting of any digital solution. Future research will consider how this digital programme can be used intelligently within a home environment i.e. smart home-based monitoring and assessment via the toolkit.

\section{ACKNOWLEDGEMENT}

The authors would like to thank all participants contributing to the participatory design sessions. This research is part of a Higher Education and Innovation Funding (HEIF) project called "Creating a FACETS digital toolkit to promote quality of life of people with multiple sclerosis". Further details: http://research.bournemouth.ac.uk/ project/msresearch-at-bournemouth-university/

\section{REFERENCES}

Clegg, D. and Barker, R. (1994). Case method fasttrack: a RAD approach. Addison-Wesley Longman Publishing Co., Inc.

Kanavos, P., Tinelli, M. and Efthymiadou, O. (2016) Towards better outcomes in multiple sclerosis by addressing policy change: The International MultiPIE Sclerosis Study (IMPrESS). The London School of Economics and Political Science, London. 
Lawlor-Savage, L. and Prentice, J. L. (2014) Digital cognitive behaviour therapy (CBT) in Canada: Ethical considerations. Canadian Psychology / Psychologie Canadienne, 55(4), 231-239.

Pulman, A., Collard, S., Miller, J., Thomas, P., Jiang, N., Dogan, H., Kersten, P., Gay, M., Davies Smith, A., Hourihan, S., Roberts, F., Stanley, K., Pretty, K. and Thomas, S. (2018) Consultation on delivering an MS-fatigue management group programme in an online format (cFACETS). Bournemouth University Clinical Research Unit, Bournemouth.

Thomas, P.W., Thomas, S., Kersten, P., Jones, R., Nock, A., Slingsby, V., Green, C., Baker, R., Galvin, K. and Hillier, C. (2010) Multi-centre parallel arm randomised controlled trial to assess the effectiveness and cost-effectiveness of a group-based cognitive behavioural approach to managing fatigue in people with multiple sclerosis. BMC Neurology 10(1), 43.

Thomas, P.W., Thomas, S., Kersten, P., Jones, R., Slingsby, V., Nock, A., Smith, A.D., Baker, R., Galvin, K.T. and Hillier, C. (2014). One year follow-up of a pragmatic multi-centre randomised controlled trial of a group-based fatigue management programme (FACETS) for people with multiple sclerosis. BMC Neurology 4(1), 109.

Thomas, S., Thomas, P.W., Kersten, P., Jones, R., Green, C., Nock, A., Slingsby, V., Davies, S.A., Baker, R., Galvin, K.T. and Hillier, C. (2013) A pragmatic parallel arm multi-centre randomised controlled trial to assess the effectiveness and cost-effectiveness of a group-based fatigue management programme (FACETS) for people with multiple sclerosis. Journal of Neurology and Neurosurgery Psychiatry 84:1092-9.

Thomas, S., Thomas, P.W., Nock, A., et al. (2010) Development and preliminary evaluation of a cognitive behavioural approach to fatigue management in people with multiple sclerosis. Patient Education and Counseling 78, 240-249. 